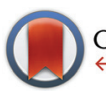

CrossMark

Cite this: Food Funct., 2017, 8, 241

\title{
Effects of liquid oil vs. oleogel co-ingested with a carbohydrate-rich meal on human blood triglycerides, glucose, insulin and appetite
}

\author{
Sze-Yen Tan, ${ }^{a}$ Elaine Wan-Yi Peh, ${ }^{a}$ Alejandro G. Marangoni ${ }^{\mathrm{b}}$ and \\ Christiani Jeyakumar Henry*a,c
}

\begin{abstract}
We examined the differences in how coconut oil in a liquid or oleogel form affects blood triglycerides, glucose, insulin, and appetite when co-ingested with a carbohydrate-rich meal. This was a randomised, controlled, crossover study where eligible participants attended a screening visit where baseline demographics were measured. On test days, participants arrived at the laboratory after an overnight fast of 10 hours. Upon arrival, cannulation of the antecubital vein was performed and fasting capillary blood glucose, plasma insulin and triglyceride levels, and appetite sensations were measured. Following that, orange juice and rice porridge alone (control), or with $22.25 \mathrm{~g}$ of coconut oil (CO) or $25 \mathrm{~g}$ of coconut oleogel (CG) (22.25 g coconut oil $+2.75 \mathrm{~g}$ ethylcellulose to form an oleogel) was consumed. Subsequently, capillary blood glucose and plasma insulin and triglyceride levels were measured at fixed intervals for 6 hours. Appetite sensations were also measured using visual analog scales every 30 minutes. Sixteen healthy young adult males completed the study (age $=27 \pm 6$ years, weight $=65.5 \pm 5.5 \mathrm{~kg}$, BMI $=21.9 \pm 1.7 \mathrm{~kg} \mathrm{~m}^{-2}$ ). After test meals, glucose, insulin, triglycerides and appetite sensations changed significantly (time effects, $p<0.001$ ). Significant time $x$ treatment effects were found only in postprandial glucose $(p=0.015)$ and triglyceride $(p=0.001)$ changes. CO reduced the peak of the glucose response, and increased the incremental area under the curve for postprandial triglycerides. CG produced outcomes comparable to those of the control treatment. Appetite sensations did not differ between all treatments. The gelling of coconut oil with ethylcellulose into an oleogel form reversed its effects on postprandial glucose and triglycerides.
\end{abstract}

Received 29th August 2016. Accepted 19th November 2016

DOI: $10.1039 /$ c6fo01274d

www.rsc.org/foodfunction cholesterol and blood triglycerides (TGs). LDL cholesterol is a well-established independent predictor of cardiovascular disease, ${ }^{4,5}$ and it is elevated during the digestion, transportation, and metabolism of dietary fats especially saturated fats. ${ }^{6}$ Saturated fats have also been shown to increase blood TG concentrations, ${ }^{7}$ which subsequently suppresses LDL-cholesterol receptor activities in the liver and hinders the clearance of LDL cholesterol from circulation. Prolonged intravascular residence time of LDL cholesterol increases its artherogenic potential. ${ }^{8}$ Hence, this emphasises the importance of managing TG levels in order to reduce the risk of cardiovascular disease.

Besides cardiovascular disease risks, saturated fats have also been shown to induce insulin resistance in humans, ${ }^{9}$ and they exhibit lower appetite-suppressing effects compared to unsaturated fats. ${ }^{10}$ Given the adverse effects of saturated fats, the reduction or replacement of coconut oil seems to be logical and essential. However, such strategies may be challenging as coconut oil imparts a unique flavour that is a part of traditional Asian cuisines and is often more affordable than other oils. An alternative and novel approach to minimise the
${ }^{a}$ Clinical Nutrition Research Centre (CNRC), Singapore Institute for Clinical Sciences (SICS), Agency for Science, Technology and Research (A*STAR), Singapore. E-mail: Jeya_Henry@sics.a-star.edu.sg; Fax: +65 6776 6840; Tel: +65 64070793 ${ }^{b}$ Department of Food Science, University of Guelph, Guelph, Canada

${ }^{c}$ Department of Biochemistry, Yong Loo Lin School of Medicine, National University of Singapore, Singapore 
potential and putative adverse effects of coconut oil without eliminating it entirely from a diet is to consume it in a different physical form such as an oleogel. Oleogel is a semisolid gel-like structure of dietary fat that is formed when ethylcellulose is dissolved into liquid vegetable oil at approximately $150{ }^{\circ} \mathrm{C}$, and a polymer network is formed as the liquid oil returns to room temperature. Through this process, the physical form of liquid oil is altered. Although the physical properties of ethylcellulose-based oleogels have been widely studied and published in the food science literature, how dietary fats in this form affect human health is yet to be thoroughly investigated. A recently in vitro study has demonstrated that the transformation of liquid oil to an oleogel reduced the digestibility of an oleogel substantially and hence limits the bioaccesibility of lipids, ${ }^{11}$ implying that coconut oil in an oleogel form may reduce its adverse health effects.

While limiting the digestibility of an oleogel may appear to be beneficial in terms of metabolic health, it presents a new challenge as dietary fats have also been shown to play an important role in postprandial glycemia regulation. Our previous study documented that the addition of oil to a carbohydrate-rich meal significantly reduced glycemic response. ${ }^{12}$ One potential explanation for this observation is that free-fatty acids, released from fat digestion in the intestine, stimulate the release of several gut hormones such as cholecystokinin and peptide-YY which reduce gastric emptying rates. ${ }^{13-15}$ This phenomenon, also known as ileal-brake, in turn reduces glucose release from the stomach and hence results in a slower rate of absorption of glucose into the circulatory system. ${ }^{16}$ Managing postprandial blood glucose concentrations after carbohydrate-rich high glycemic index meals, which are common in Asian diets, is important as postprandial hyperglycemia (rapid increase and high blood glucose peak) has adverse effects on metabolic health. ${ }^{17,18}$ At the same time, lower gastric emptying rates translate into increased gastric distention, which is critical in promoting satiation and satiety. ${ }^{19}$ Finding a balance is essential in order to achieve optimal physiological responses after consuming coconut oleogel i.e. lower effects on postprandial TG but sufficient fatty acids to reduce glycemia are of utmost importance. In this study, we aimed to quantify the effects of coconut oil in a liquid form or as an oleogel added to a carbohydrate-rich meal on postprandial TG, glucose, insulin, and appetitive responses.

\section{Methods}

\section{Study design}

This was a randomised, controlled, crossover study where participants attended a screening visit followed by three test sessions.

\section{Study population}

Healthy male participants were recruited from the general public by means of advertisements, flyers, and personal communications. Only males were included to eliminate potential variations due to hormonal changes during menstrual cycles in women. The inclusion criteria were: body weight $\geq 45 \mathrm{~kg}$; body mass index (BMI) 18-25 $\mathrm{kg} \mathrm{m}^{-2}$; blood pressure $\leq 140 /$ $90 \mathrm{mmHg}$; and normal fasting blood glucose $\left(<6.0 \mathrm{mmol} \mathrm{L}^{-1}\right)$. People with major chronic disease such as heart disease, cancer or diabetes mellitus, family history of diabetes or familial hypercholesterolemia, history of liver and renal problems, intolerances or allergies to test products, and those who are taking insulin or drugs known to affect glucose metabolism and body fat distribution were excluded from the study. Based on the recommendations by the $\mathrm{FAO} / \mathrm{WHO}$, a minimum of 10 subjects are required during the analysis of glycemic, insulinemic and lipidemic responses in humans to take into account the inter-individual variations. Hence, a sample size of 15 was deemed adequate for the current study.

\section{Experimental protocol}

Volunteers who expressed interest and met all inclusion criteria attended a screening session in the morning after an overnight fast, where written informed consent was obtained from the participants. The blinding of participants to different oil forms was not possible in this study, but the participants were told that the purpose of our study was to investigate the health effects of different breakfast meals. During the screening session, anthropometric measurements including height, weight and body fat percentage were taken, health and physical activity (the International Physical Activity Questionnaire) ${ }^{20}$ questionnaires were completed, and blood pressure was measured after a brief resting period of five minutes. Finally, fasting blood glucose concentration was determined via finger prick capillary blood samples to confirm normal fasting blood glucose. On the test days, participants arrived at the laboratory in the morning following a 10 hour overnight fast. Upon arrival, participants rested briefly before the baseline satiety level was measured using validated visual analog scales (VAS), ${ }^{21}$ and an indwelling catheter was inserted into the participants' antecubital vein, which was kept patent by flushing with $3 \mathrm{ml}$ non-heparinised saline. Venous blood samples $(5 \mathrm{ml})$ were collected after the insertion of the catheter and a baseline finger-prick was also obtained to determine baseline fasting capillary blood glucose. Capillary blood glucose was chosen because tissues consume glucose, which makes venous blood glucose levels lower and more varied (higher coefficient of variation) than arterial blood. ${ }^{22}$ After the fasting assessments, a test meal (breakfast) was provided to participants, which was consumed by participants within 10 minutes in an upright sitting position. The order of food item consumption within the breakfast meal was not standardised as long as test foods were consumed within the given time. After the test meal, participants rated their liking of the test meals and $5 \mathrm{ml}$ of venous blood samples were taken at 10, 20, 30, 45, 60, 90, $120,150,180,210,240,270,300,330$ and 360 minutes to determine plasma insulin and triglyceride (TG) concentrations. The six-hour study period was selected because this latency was required to observe maximal postprandial plasma TG concentrations. ${ }^{23}$ The participants were encouraged to minimise physical activity during the test period. The study 
session terminated after six hours, and participants repeated the study sessions (with one week washout) until all test meals were completed. The study was approved by the Domain Specific Review Board (DSRB) of the National Healthcare Group (Reference Number: 2015/01098) and registered under ClinicalTrials.gov (reference number: NCT02875275).

\section{Test meals}

Subjects received three different test meals in a random order, which consisted of: (1) instant rice porridge (Knorr chicken and mushroom cup porridge, Unilever Singapore Pte. Ltd, Singapore) and orange juice (Marigold 100\% Juice, Malaysia Dairy Industries Pte. Ltd, Singapore) only (control group), (2) $22.25 \mathrm{~g}$ liquid coconut oil (Nutiva virgin organic coconut oil, Nutiva Inc., Richmond USA) added to the porridge (CO group) and orange juice, and (3) $25 \mathrm{~g}$ coconut oleogel added to the instant rice porridge (CG group) and orange juice. In the CG treatment, the coconut oleogel was cut into small pieces so that they could be incorporated into the rice porridge evenly. The majority of fatty acids found in coconut oil and the macronutrient compositions of the test meals are shown in Table 1. The coconut oleogel was prepared by dissolving $11 \% \mathrm{w} / \mathrm{w}$ ethylcellulose (viscosity $45 \mathrm{cP}$ ) and $0.01 \% \mathrm{w} / \mathrm{w}$ butylated hydroxytoluene (as antioxidant) to coconut oil at $140-150{ }^{\circ} \mathrm{C}(22.25 \mathrm{~g}$ coconut oil $+2.75 \mathrm{~g}$ ethylcellulose). Once the ethylcellulose was completely dissolved in the coconut oil, it was allowed to return to room temperature to form an oleogel. The details of the oleogel preparation have been previously described. ${ }^{24}$

\section{Measures}

During the screening session, height was measured using a stadiometer (Seca Limited, Birmingham, West Midlands,

Table 1 Macronutrient composition and serving sizes of the test meals and the fatty acid composition of coconut oil

\begin{tabular}{|c|c|c|c|}
\hline & Control & $\mathrm{CO}$ & CG \\
\hline Energy, kJ & 905 & 1740 & 1740 \\
\hline Carbohydrates, $\mathrm{g}$ & 46.2 & 46.2 & 46.2 \\
\hline Total fat, $\mathrm{g}$ & 1.3 & 23.6 & 23.6 \\
\hline Total protein, $\mathrm{g}$ & 4.2 & 4.2 & 4.2 \\
\hline Test meals & $\begin{array}{l}\text { Instant rice } \\
\text { porridge }(285 \mathrm{~g})+ \\
\text { orange juice } \\
(250 \mathrm{ml})\end{array}$ & $\begin{array}{l}\text { Control meal } \\
+ \text { coconut oil } \\
(22.25 \mathrm{~g})\end{array}$ & $\begin{array}{l}\text { Control meal } \\
+ \text { coconut } \\
\text { oleogel } \\
(25 \mathrm{~g})^{a}\end{array}$ \\
\hline \multicolumn{4}{|c|}{ Major fatty acids of coconut oil (per $100 \mathrm{~g}$ ) } \\
\hline C8:0 (saturated) & \multicolumn{3}{|c|}{$6.8 \mathrm{~g}$} \\
\hline C10:0 (saturated) & \multicolumn{3}{|l|}{$5.4 \mathrm{~g}$} \\
\hline C12:0 (saturated) & \multicolumn{3}{|l|}{$41.8 \mathrm{~g}$} \\
\hline C14:0 (saturated) & \multicolumn{3}{|l|}{$16.7 \mathrm{~g}$} \\
\hline C16:0 (saturated) & \multicolumn{3}{|l|}{$8.6 \mathrm{~g}$} \\
\hline C18:0 (saturated) & \multicolumn{3}{|l|}{$2.5 \mathrm{~g}$} \\
\hline $\begin{array}{l}\text { C18:1 } \\
\text { (monounsaturated) }\end{array}$ & \multicolumn{3}{|l|}{$6.3 \mathrm{~g}$} \\
\hline $\begin{array}{l}\text { C18:2 n-6 } \\
\text { (polyunsaturated) }\end{array}$ & \multicolumn{3}{|l|}{$1.7 \mathrm{~g}$} \\
\hline
\end{tabular}

CO - coconut oil treatment; CG - coconut oleogel treatment. ${ }^{a}$ The oleogel contained $22.25 \mathrm{~g}$ coconut oil and $2.75 \mathrm{~g}(11 \% \mathrm{w} / \mathrm{w})$ of ethylcellulose $45 \mathrm{cP}$ viscosity.
Middlesex, UK), body weight and body composition was measured using an 8-electrode bioelectrical impedance analysis (BIA) device (Tanita BC-418, Tokyo, Japan), blood pressure was measured using an Omron blood pressure monitor (Model HEM-907) at baseline. Blood glucose levels (screening session and test visits) were measured using the HemoCue $201^{+}$ Glucose RT analyser (HemoCue Ltd, Dronfield, UK). The Hemocue is a reliable method that has intra- and inter-assay CVs of $1.2 \%$ and $1.3 \%$, respectively, for capillary blood glucose analysis, and it is an accepted method for blood glucose assessments by the $\mathrm{FAO} / \mathrm{WHO}{ }^{25}$ On test days, two baseline finger prick blood samples were taken 5 minutes apart to measure fasting glucose levels, and subsequent finger pricks were taken at fixed intervals described in the study protocol. For each finger prick, the first two drops of expressed blood were discarded and the next drop was collected directly into a microcuvette for blood glucose analysis. Postprandial blood glucose concentration changes were determined by computing the difference in blood glucose concentration between fasting and particular time points. Changes in blood glucose were then used to calculate the incremental area under the curve (iAUC) using a trapezoidal rule for the entire 360 minute testing period, ignoring the area beneath the baseline, to obtain the glycemic response. ${ }^{22,26}$ To determine insulin and TG responses to test meals, venous blood samples were collected at fixed intervals in vacutainers with disodium EDTA, centrifuged, and plasma was then aliquoted and stored at $-80^{\circ} \mathrm{C}$ until analysis was carried out. Plasma insulin and TG concentrations were measured using an immunochemistry analyser COBAS e411 (Roche, HITACHI, USA) and COBAS c311 (Roche, HITACHI, USA), respectively. The intraand inter-assay CVs are $<5 \%$ and $<6 \%$ respectively for insulin, and $<1.5 \%$ and $<2 \%$ respectively for TG. Changes from the baseline and iAUC were also calculated for insulin and TG responses. The liking of the test meals was assessed using a $200 \mathrm{~mm}$ VAS anchored at 'dislike extremely' $(-100 \mathrm{~mm})$, 'neither like nor dislike' $(0 \mathrm{~mm})$, and 'like extremely' $(+100 \mathrm{~mm})$. Appetite sensations were measured using validated $100 \mathrm{~mm}$ VAS $^{27}$ before and after consuming the test food, and every 30 minutes subsequently. The appetite-rating questionnaire included questions on hunger, desire to eat, prospective consumption and fullness.

\section{Statistical analysis}

Participants' baseline demographics and all variables are expressed as the mean \pm standard deviation unless otherwise specified. Within and between-treatment comparisons of the means between test days for all variables were performed using the general linear model for repeated measures ANOVA with Bonferroni corrections. Tukey's post hoc analyses were conducted to compare the differences between treatments at all time points and reported accordingly. All statistical analyses were performed using the Statistical Package for the Social Sciences software (IBM SPSS version 23.0, IBM Corp., Armonk. NY, USA) and statistical significance was set at $p<0.05$, twotailed. 


\section{Results}

Sixteen adult males met the study inclusion criteria and completed the study. At baseline, participants were: age $=27 \pm 6$ years, weight $=65.5 \pm 5.5 \mathrm{~kg}, \mathrm{BMI}=21.9 \pm 1.7 \mathrm{~kg} \mathrm{~m}^{-2}$, body fat $=15.4 \pm 3.1 \%$, and fasting blood glucose $=4.5 \pm 0.5 \mathrm{mmol} \mathrm{L}^{-1}$. At the beginning of each test visit, fasting glucose, insulin and TG were not significantly different $(p=0.660, p=0.535$ and $p=$ 0.800 , respectively). The liking scores did not differ significantly between test meals: control $=26 \pm 44 \mathrm{~mm}, \mathrm{CO}=14 \pm$ $33 \mathrm{~mm}$ and $\mathrm{CG}=9 \pm 27 \mathrm{~mm}(p=0.192)$. Changes in blood glucose, insulin and triglycerides from the baseline as well as the iAUC of these parameters are presented in Fig. 1. Significant time effects were found in all variables $(p<0.05)$, but the interaction (time $\times$ treatment) effects were significant in postprandial TG $(p=0.001)$ and glucose $(p=0.015)$ only (significantly lower in CO during the first 60 minutes). The iAUC for TG was significantly different between study groups ( $p$ $=0.013$ ), which was due to a significantly higher iAUC in CO compared to the other two groups. Over 360 minutes, time effects were found with hunger, fullness, desire-to-eat, and prospective eating scores (all $p<0.001$ ), but none of these parameters showed significant interaction effects (hunger, $p=$ 0.874 , fullness, $p=0.743$, desire-to-eat, $p=0.915$, and prospective eating, $p=0.981$ ). The postprandial changes in these appetitive parameters are shown in Fig. 2. Although the postprandial excursion of fullness may appear lower in the CG group, the ratings were not significantly different between treatments at any time point throughout the experiment (Fig. 2B).

\section{Discussion}

The purpose of this study was to investigate the physiological and appetitive effects of co-ingesting coconut oil in liquid or oleogel forms together with a carbohydrate-rich meal. The reason for including a carbohydrate-rich porridge breakfast in our study was to mimic Asian diets that are relatively high in carbohydrates. The appetite ratings did not differ significantly between the control, CO and CG test meals. Our findings were consistent with previous studies, where the addition of dietary fat to a meal was shown to have minimal effects on human appetite and food intake acutely. ${ }^{28,29}$ Although foods in a solid form have been shown to be more satiating due to the extra mechanical chewing efforts and longer oral processing time of solid foods, ${ }^{30-32}$ the addition of a semisolid oleogel to a meal did not show additional appetitive benefits. The lack of anticipated appetite-suppressing effects of the oleogel in our study may be due to the small volume of oleogel (relative to the entire breakfast meal) ingested. Although the oleogel used in our study was cut up into smaller pieces, oral processing effort was still required and this process was expected to increase the digestibility of the oleogel and the bioaccesibility of fats from the oleogel polymer network structure. ${ }^{33}$ However, this was not the case based on the pattern of postprandial TG excursion observed in this study. It has been demonstrated that dietary fats are entrapped in a polymer network of ethylcellulose which delays their digestion. ${ }^{34}$ The low digestibility of the oleogel and the slower release of fatty acids from the oleogel may have prevented the effects of oil on gastric emptying, postprandial glucose, insulin, and appetite. ${ }^{35,36}$

Blood glucose changes following the ingestion of a carbohydrate-rich meal alone or with two different forms of fats resulted in significantly different glucose excursions (interaction effects, $p=0.015$ ). Blood glucose was found to elevate in smaller magnitudes at 30 and 45 minutes after CO than the control and the CG treatments. However, because the postprandial glucose in the CO group took longer to return to baseline, the iAUC of glucose was comparable across all treatments. This was consistent with findings from another study, where the coingestion of up to $30 \mathrm{~g}$ of fat with a carbohydrate-rich food did not result in glucose iAUC differences. ${ }^{37}$ Insulin release also followed similar trends, but the smaller increase observed in $\mathrm{CO}$ at 30 and 45 minutes after the coconut oil treatment failed to reach statistical significance. Together, the glucose and insulin responses appear to suggest that lower plasma glucose levels after CO could mainly be attributed to the slower emptying of glucose into the small intestine and subsequently lower absorption into circulation. Slower gastric emptying rate of glucose into the intestine by CO is further supported by lower insulin release, which suggests lower detection of glucose in the intestine. A previous human study has also reported that the addition of fat into a meal reduces the rates of gastric emptying and blunted postprandial glucose and insulin levels. ${ }^{38}$

More interestingly, the CG treatment was found to produce differential effects compared to the CO treatment even though these test meals were matched in energy and total fat content. The postprandial glucose and insulin patterns (Fig. 1A and B, line graphs) after CG were almost identical to the glucose and insulin responses induced by the control meal. A potential explanation of this 'lack-of-effect' in the CG treatment can be drawn from the TG response patterns after the CG test meal. A significantly important observation is shown in Fig. 1C, where postprandial TG levels were found to elevate significantly in the $\mathrm{CO}$ group, but not the control and the CG groups. As far as we are aware, this is the first study that demonstrates that co-ingestion of coconut oil as an oleogel with a carbohydrate source completely attenuates the TG excursion seen when coconut oil is administered in a form of liquid. Although a previous study reported that the consumption of canola oil in a gel form increased postprandial TG as much as liquid canola oil, it should be noted that the gel used in this study was prepared using 12-hydroxystearic acid, ${ }^{23}$ which has physical and chemical structures that are markedly different from oleogels made with ethylcellulose. ${ }^{39}$ It is possible that the coconut oil in a liquid form was digested and absorbed into circulation more readily than the coconut oleogel where the fats are trapped in a complex ethylcellulose polymer network that resists digestion. ${ }^{11}$ The TG concentrations after the CO and CG test meals both peaked and then declined at about 240 and 270 minutes, which suggests that the oleogel may have resisted digestion and hence produced lower concentrations instead of delayed appearance of TG in the circulation. 

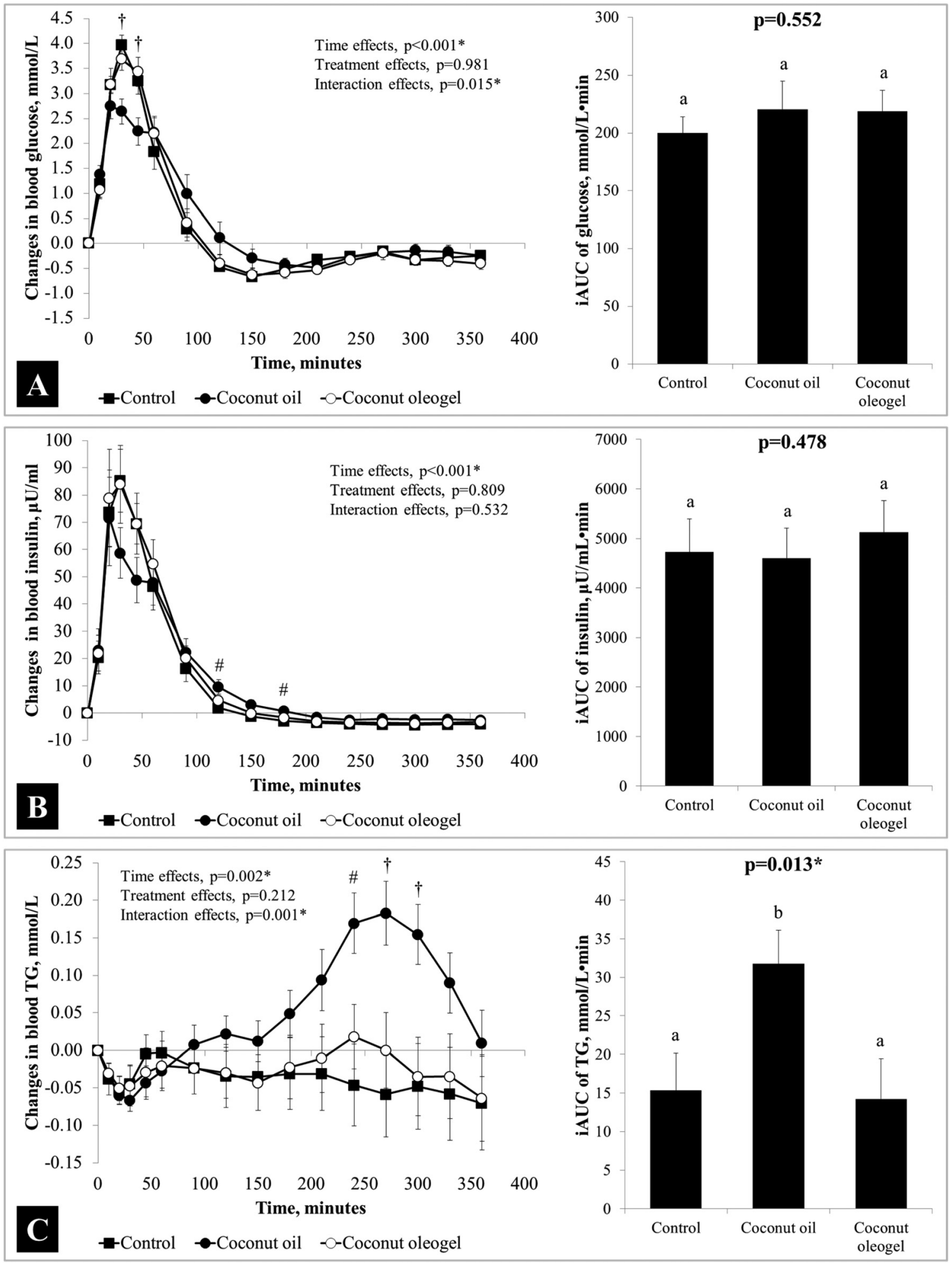

Fig. 1 Postprandial changes in glucose (A), insulin (B), and triglycerides (TG) (C) following the consumption of a carbohydrate-rich breakfast alone or with coconut oil or coconut oleogel. Significant interaction (time $\times$ treatment) effects were found in postprandial glucose and TG. \# indicates significant differences between coconut oil (CO) vs. control only, while $\dagger$ indicates significant differences between coconut oil vs. the other two treatments (control and coconut oleogel). Bar graphs represent the incremental area under the curve (iAUC) over 360 minutes for corresponding blood biochemical indices. Bars with different superscript letters were significantly different $(p<0.05)$. A significant difference was found in the iAUC for triglycerides due to the higher iAUC following coconut oil co-ingestion with the meal. 


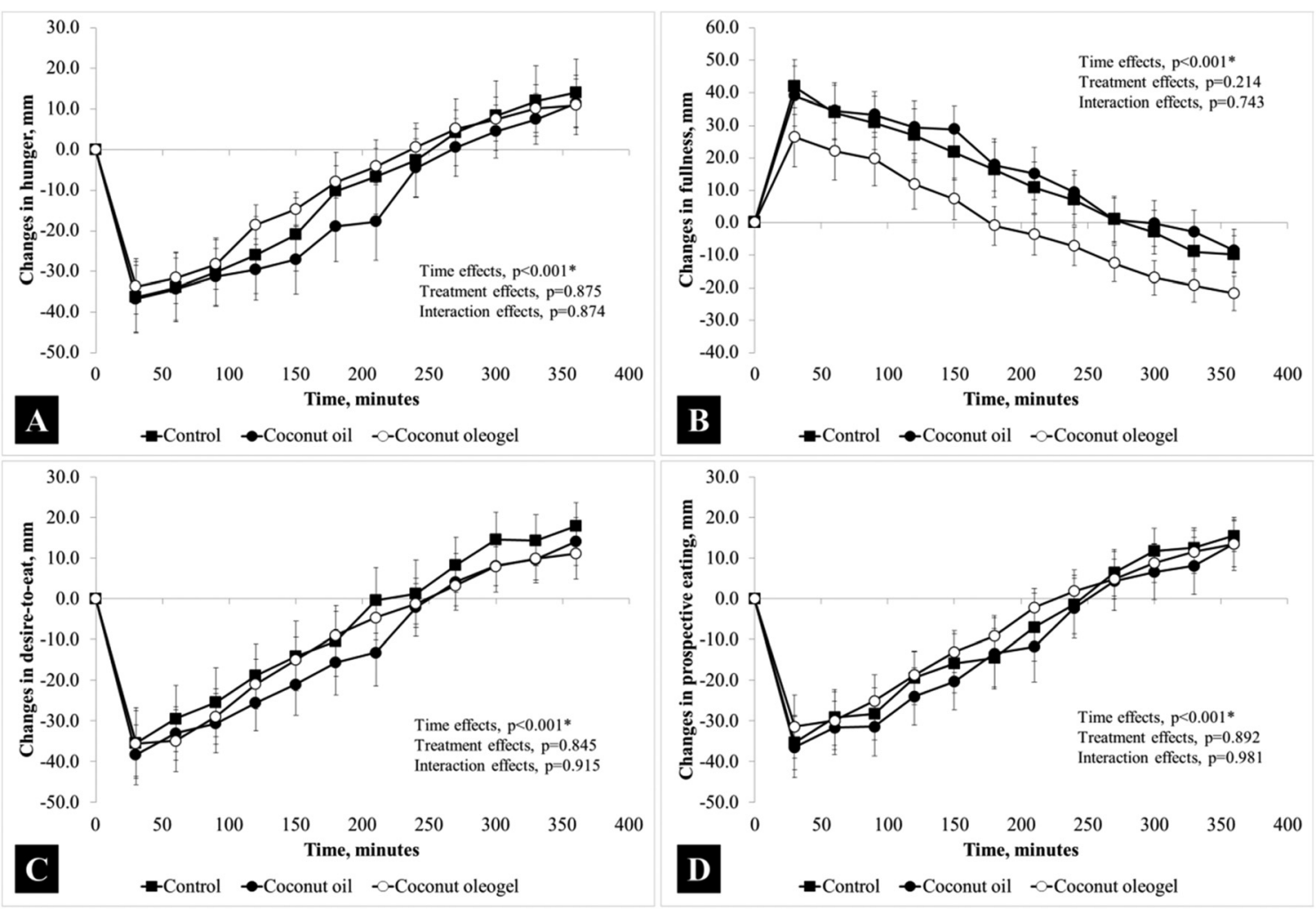

Fig. 2 Postprandial changes in hunger (A), fullness (B), desire-to-eat (C), and prospective eating (D) ratings following the consumption of a carbohydrate-rich breakfast alone or with coconut oil or coconut oleogel. Significant time effects were found in all aspects of the appetite ratings (all $p<0.001$ ), but no significant effects were observed between treatments overall, or at any time point.

The observed differences in digestion behavior between coconut oil and coconut oleogel can be explained from the structure of the ethylcellulose oleogel. The oleogel used in this study was prepared with ethylcellulose with a molecular weight of $45 \mathrm{cP}, 11 \% \mathrm{w} / \mathrm{w}$ quantity, and gelled at a higher temperature of $140-150{ }^{\circ} \mathrm{C}$. When prepared under these conditions, oleogels have been reported to require a higher back extrusion force at maximum penetration, ${ }^{40}$ implying that the oleogel is firmer and harder to breakdown by mechanical chewing. Oil leakage from the oleogel was also found to be negligible over 12 days, ${ }^{23}$ suggesting that the oil was very well-encapsulated by the polymer network and not freely available. Recent experiments have demonstrated how both in vitro lipolysis and betacarotene transfer from ethylcellulose oleogels were significantly lower in ethylcellulose oleogels prepared with higher molecular weight versions of ethylcellulose. ${ }^{11,41}$ Previous experiments also demonstrated how the hardness of ethylcellulose oleogels were controlled by three factors, polymer concentration, polymer molecular weight and solvent quality (type of oil). ${ }^{42}$ An increase in the ethylcellulose concentration leads to a decrease in gel cell size within the ethylcellulose oleogels. Oil is physically trapped within these cells and a decrease in cell size leads to a greater amount of entrapped oil dispersion and an increase in mechanical strength of the gels. However, another factor that influenced strength was ethylcellulose molecular weight, and this increase in hardness was not accompanied by a decrease in cell size. We speculated that the observed increase in gel hardness was due to an increase in the elastic modulus of the ethylcellulose walls within the oleogel. Previous studies also reported that increases in ethylcellulose concentration for low molecular weight ethylcellulose $10 \mathrm{cP}$ resulted in an increase in gel hardness but did not lead to a decrease in TG lipolysis or beta carotene transfer (relative to free beta carotene loaded oil). ${ }^{11,41}$ However, a pronounced increase in gel hardness and a decrease in TG lipolysis and beta carotene transfer were observed when higher molecular weight ethylcellulose $(45 \mathrm{cP})$ was used to gel the oil. So, the key factor affecting the rate of lipolysis of the ethylcellulose oleogels is the mechanical strength of the ethylcellulose walls, and not necessarily the size of the cells within the gels. The physical characteristics of ethylcellulose polymers have been reported previously. ${ }^{43} \mathrm{~A}$ number of other studies that modified the structure of dietary oils using different techniques such as monoglyceride-structured emulsification have also shown that the encapsulation of oil through a mesoscopic structure was successful in reducing the TG response in blood. ${ }^{44,45}$ Therefore, it is reasonable to speculate that the near-identical outcomes between the control and the coconut oleogel were due to lower digestibility of the oleogel. However, since we did not measure fat digestibility in this study, verification using in vitro or in vivo digestion models is warranted in the future. 
Thus, we can conclude from in vitro studies and our current work that the large decrease in TG serum accumulation observed for coconut oleogels vs. liquid coconut oil was due to an encapsulation effect of the coconut oil within a "closedcell" ethylcellulose gel with very strong walls. These walls are strong enough to survive the shear forces encountered in the gastrointestinal tract and to physically prevent pancreatic lipase from reaching the dietary fats. This points to a novel physical strategy to mitigate serum TG overloads after acute fatty meal ingestions and the associated negative physiological effects. It also points to interesting future work where quantitative relationships could be established between cell wall strength and bioavailability and bioaccesibility in ethylcellulose-based delivery systems.

Our study has some limitations. First, the study was conducted in a small group of healthy young Chinese males so the generalisability of our findings to other populations e.g. people with abnormal blood glucose needs to be examined in the future. Second, our sample population size was modest, however the within-subject crossover design reduced the between-subject variability in our study. Third, gastric emptying rates were not measured so we were not able to confirm our hypothesis that lower blood glucose and insulin in the liquid oil treatment was due to this factor. This should be investigated in the future. Finally, we did not measure other insulinotropic hormones because this study was designed to be an exploratory study and the exclusion of these measurements was due to budget constraints.

\section{Conclusion}

A novel observation of our study was the significant reduction in postprandial TG excursion when coconut oil was consumed in an oleogel form. Our trial is the first study that documents such a transformation in TG response when coconut oil is consumed as conventional oil or as a coconut oleogel together with a carbohydrate-rich meal. Given the importance of increased TG and cholesterol as biomarkers of cardiovascular risks, our observations have major biomedical and public health implications, especially in Asia where coconut oil remains a major vegetable based oil. The ability to modulate the physiological response of coconut oil by manipulating the oil into an oleogel opens up a novel area of food science and nutrition whereby oils that have an intrinsic attribute in increasing TG may be transformed into oleogel based oils that have little or no impact on postprandial TG. Future work will incorporate coconut oleogel into different food matrices, and test the timing of oleogel consumption to maximise its effects on physiological responses.

\section{Authorship}

All authors contributed to the study design, data analysis and interpretation, and the writing of the manuscript.

\section{Conflict of interests}

The authors declare no conflict of interests.

\section{Acknowledgements}

This study was funded by the Agency for Science, Technology and Research ( $A^{*}$ STAR). The coconut oil and oleogel used in this study was kindly prepared and provided by Professor Alejandro Marangoni from the University of Guelph, Canada. We would also like to acknowledge assistance from Susanna Lim, Joseph Lim and Kevin Tan.

\section{Notes and references}

1 M. B. Katan, P. L. Zock and R. P. Mensink, Effects of fats and fatty acids on blood lipids in humans: an overview, Am. J. Clin. Nutr., 1994, 60, 1017S-1022S.

2 M. Guasch-Ferré, N. Babio, M. A. Martínez-González, D. Corella, E. Ros, S. Martín-Peláez, R. Estruch, F. Arós, E. Gómez-Gracia and M. Fiol, Dietary fat intake and risk of cardiovascular disease and all-cause mortality in a population at high risk of cardiovascular disease, Am. J. Clin. Nutr., 2015, 102, 1563-1573.

3 Y. Li, A. Hruby, A. M. Bernstein, S. H. Ley, D. D. Wang, S. E. Chiuve, L. Sampson, K. M. Rexrode, E. B. Rimm and W. C. Willett, Saturated fats compared with unsaturated fats and sources of carbohydrates in relation to risk of coronary heart disease: a prospective cohort study, J. Am. Coll. Cardiol., 2015, 66, 1538-1548.

4 B. V. Howard, D. C. Robbins, M. L. Sievers, E. T. Lee, D. Rhoades, R. B. Devereux, L. D. Cowan, R. S. Gray, T. K. Welty and O. T. Go, LDL cholesterol as a strong predictor of coronary heart disease in diabetic individuals with insulin resistance and low LDL the Strong Heart Study, Arterioscler., Thromb., Vasc. Biol., 2000, 20, 830-835.

5 V. Manninen, L. Tenkanen, P. Koskinen, J. Huttunen, M. Mänttäri, O. Heinonen and M. Frick, Joint effects of serum triglyceride and LDL cholesterol and HDL cholesterol concentrations on coronary heart disease risk in the Helsinki Heart Study. Implications for treatment, Circulation, 1992, 85, 37-45.

6 K. K. Berneis and R. M. Krauss, Metabolic origins and clinical significance of LDL heterogeneity, J. Lipid Res., 2002, 43, 1363-1379.

7 H. N. Ginsberg, P. Kris-Etherton, B. Dennis, P. J. Elmer, A. Ershow, M. Lefevre, T. Pearson, P. Roheim, R. Ramakrishnan and R. Reed, Effects of reducing dietary saturated fatty acids on plasma lipids and lipoproteins in healthy subjects The Delta Study, Protocol 1, Arterioscler., Thromb., Vasc. Biol., 1998, 18, 441-449.

8 D. K. Spady and J. M. Dietschy, Dietary saturated triacylglycerols suppress hepatic low density lipoprotein receptor 
activity in the hamster, Proc. Natl. Acad. Sci. U. S. A., 1985, 82, 4526-4530.

9 C. Xiao, A. Giacca, A. Carpentier and G. Lewis, Differential effects of monounsaturated, polyunsaturated and saturated fat ingestion on glucose-stimulated insulin secretion, sensitivity and clearance in overweight and obese, non-diabetic humans, Diabetologia, 2006, 49, 1371-1379.

10 C. L. Lawton, H. J. Delargy, J. Brockman, F. C. Smith and J. E. Blundell, The degree of saturation of fatty acids influences post-ingestive satiety, Br. J. Nutr., 2000, 83, 473-482.

11 C. O'Sullivan, In-vitro bioaccessibility and stability of betacarotene in ethylcellulose oleogels, Masters thesis, University of Guelph, 2016.

12 E. Lau, W. Zhou and C. Henry, Effect of fat type in baked bread on amylose-lipid complex formation and glycaemic response, Br. J. Nutr., 2016, 115, 2122-2129.

13 P. W. Maljaars, T. Symersky, B. C. Kee, E. Haddeman, H. P. Peters and A. A. Masclee, Effect of ileal fat perfusion on satiety and hormone release in healthy volunteers, Int. J. Obes., 2008, 32, 1633-1639.

14 P. Maljaars, H. Peters, D. Mela and A. Masclee, Ileal brake: a sensible food target for appetite control. A review, Physiol. Behav., 2008, 95, 271-281.

15 C. Feinle, T. Rades, B. Otto and M. Fried, Fat digestion modulates gastrointestinal sensations induced by gastric distention and duodenal lipid in humans, Gastroenterology, 2001, 120, 1100-1107.

16 S. Normand, Y. Khalfallah, C. Louche-Pelissier, C. Pachiaudi, J.-M. Antoine, S. Blanc, M. Desage, J. P. Riou and M. Laville, Influence of dietary fat on postprandial glucose metabolism (exogenous and endogenous) using intrinsically ${ }^{13} \mathrm{C}$-enriched durum wheat., Br. J. Nutr., 2001, 86, 3-11.

17 A. Ceriello, Postprandial hyperglycemia and diabetes complications is it time to treat?, Diabetes, 2005, 54, 1-7.

18 M. Hanefeld, C. Koehler, F. Schaper, K. Fuecker, E. Henkel and T. Temelkova-Kurktschiev, Postprandial plasma glucose is an independent risk factor for increased carotid intima-media thickness in non-diabetic individuals, Atherosclerosis, 1999, 144, 229-235.

19 K. Hveem, K. Jones, B. Chatterton and M. Horowitz, Scintigraphic measurement of gastric emptying and ultrasonographic assessment of antral area: relation to appetite, Gut, 1996, 38, 816-821.

20 M. L. Booth, B. E. Ainsworth, M. Pratt, U. Ekelund, A. Yngve, J. F. Sallis and P. Oja, International physical activity questionnaire: 12-country reliability and validity, Med. Sci. Sports Exercise, 2003, 195, 3508-1381.

21 A. Flint, A. Raben, J. E. Blundell and A. Astrup, Reproducibility, power and validity of visual analogue scales in assessment of appetite sensations in single test meal studies, Int. J. Obes., 2000, 24, 38-48.

22 F. Brouns, I. Bjorck, K. N. Frayn, A. L. Gibbs, V. Lang, G. Slama and T. M. Wolever, Glycaemic index methodology, Nutr. Res. Rev., 2005, 18, 145-171.

23 T. A. Stortz, A. K. Zetzl, S. Barbut, A. Cattaruzza and A. G. Marangoni, Edible oleogels in food products to help maximize health benefits and improve nutritional profiles, Lipid Technol., 2012, 24, 151-154.

24 M. Davidovich-Pinhas, S. Barbut and A. G. Marangoni, The gelation of oil using ethyl cellulose, Carbohydr. Polym., 2015, 117, 869-878.

$25 \mathrm{FAO} / \mathrm{WHO}$, Carbohydrates in human nutrition. Report of a Joint FAO/WHO Expert Consultation, FAO Food Nutr. Pap., 1998, 66, 1-140.

26 T. M. Wolever and D. J. Jenkins, The use of the glycemic index in predicting the blood glucose response to mixed meals, Am. J. Clin. Nutr., 1986, 43, 167-172.

27 A. Flint, A. Raben, J. E. Blundell and A. Astrup, Reproducibility, power and validity of visual analogue scales in assessment of appetite sensations in single test meal studies, Int. J. Obes. Relat. Metab. Disord., 2000, 24, 38-48.

28 J. R. Cotton, V. J. Burley, J. A. Weststrate and J. E. Bhmdell, Dietary fat and appetite: similarities and differences in the satiating effect of meals supplemented with either fat or carbohydrate, J. Hum. Nutr. Diet., 1994, 7, 11-24.

29 D. M. Woodend and G. H. Anderson, Effect of sucrose and safflower oil preloads on short term appetite and food intake of young men, Appetite, 2001, 37, 185-195.

30 J. Dhillon, C. A. Running, R. M. Tucker and R. D. Mattes, Effects of food form on appetite and energy balance, Food Qual. Prefer., 2016, 48, 368-375.

31 N. Zijlstra, R. de Wijk, M. Mars, A. Stafleu and C. de Graaf, Effect of bite size and oral processing time of a semisolid food on satiation, Am. J. Clin. Nutr., 2009, 90, 269-275.

32 C. de Graaf, Texture and satiation: the role of oro-sensory exposure time, Physiol. Behav., 2012, 107, 496-501.

33 M. M. L. Grundy, T. Grassby, G. Mandalari, K. W. Waldron, P. J. Butterworth, S. E. E. Berry and P. R. Ellis, Effect of mastication on lipid bioaccessibility of almonds in a randomized human study and its implications for digestion kinetics, metabolizable energy, and postprandial lipemia, Am. J. Clin. Nutr., 2015, 101, 25-33.

34 T. Laredo, S. Barbut and A. G. Marangoni, Molecular interactions of polymer oleogelation, Soft Matter, 2011, 7, 27342743.

35 P. W. Maljaars, H. P. Peters, D. J. Mela and A. A. Masclee, Ileal brake: a sensible food target for appetite control. A review, Physiol. Behav., 2008, 95, 271-281.

36 D. O’Donovan, M. Horowitz, A. Russo, C. Feinle-Bisset, N. Murolo, D. Gentilcore, J. Wishart, H. Morris and K. Jones, Effects of lipase inhibition on gastric emptying of, and on the glycaemic, insulin and cardiovascular responses to, a high-fat/carbohydrate meal in type 2 diabetes, Diabetologia, 2004, 47, 2208-2214.

37 E. Moghaddam, J. A. Vogt and T. M. Wolever, The effects of fat and protein on glycemic responses in nondiabetic humans vary with waist circumference, fasting plasma insulin, and dietary fiber intake, J. Nutr., 2006, 136, 25062511.

38 D. Gentilcore, R. Chaikomin, K. L. Jones, A. Russo, C. Feinle-Bisset, J. M. Wishart, C. K. Rayner and 
M. Horowitz, Effects of fat on gastric emptying of and the glycemic, insulin, and incretin responses to a carbohydrate meal in type 2 diabetes, J. Clin. Endocrinol. Metab., 2006, 91, 2062-2067.

39 A. G. Marangoni, Organogels: an alternative edible oilstructuring method, J. Am. Oil Chem. Soc., 2012, 89, 749780.

40 A. K. Zetzl, A. G. Marangoni and S. Barbut, Mechanical properties of ethylcellulose oleogels and their potential for saturated fat reduction in frankfurters, Food Funct., 2012, 3, 327-337.

41 C. O'Sullivan, S. Barbut and A. G. Marangoni, Edible oleogels for the oral delivery of lipid soluble molecules: composition and structural design considerations, Trends Food Sci. Technol., 2016, 57A, 59-73.
42 A. K. Zetzl, A. J. Gravelle, M. Kurylowicz, J. Dutcher, S. Barbut and A. G. Marangoni, Microstructure of ethylcellulose oleogels and its relationship to mechanical properties, Food Struct., 2014, 2, 27-40.

43 M. Davidovich-Pinhas, S. Barbut and A. Marangoni, Physical structure and thermal behavior of ethylcellulose, Cellulose, 2014, 21, 3243-3255.

44 J. W. Rush, P. S. Jantzi, K. Dupak, S. H. Idziak and A. G. Marangoni, Acute metabolic responses to butter, margarine, and a monoglyceride gel-structured spread, Food Res. Int., 2009, 42, 1034-1039.

45 A. G. Marangoni, S. H. Idziak, C. Vega, H. Batte, M. Ollivon, P. S. Jantzi and J. W. Rush, Encapsulation-stucturing of edible oil attenuates acute elevation of blood lipids and insulin in humans, Soft Matter, 2007, 3, 183-187. 